Capítulo 3 



\title{
La perspectiva ecofeminista: discusiones teóricas, reflexiones y tensiones ${ }^{*}$
}

\author{
Yaneth Ortiz Nova ${ }^{* *}$
}

El presente capítulo recoge las reflexiones en torno a los supuestos que desde el feminismo y la ecología configuran el ecofeminismo. Este recorrido tiene en cuenta dos elementos: por un lado, presenta la trayectoria histórica alrededor de lo que significa hablar de ecofeminismo, contextualiza el surgimiento de la categoría mujer-naturaleza y presenta las principales discusiones teóricas y políticas que se han dado al respecto. Por el otro, pretende generar una reflexión política sobre la propuesta ecofeminista latinoamericana, en un contexto en que la actividad industrial ha puesto en riesgo la vida y el avance de la sociedad hacia condiciones de justicia, igualdad y bienestar.

Hablar de la relación mujer-naturaleza exige revisar los distintos debates que se han dado al respecto y presentar diversas apuestas acerca de las razones por las cuales las mujeres han estado excluidas del debate sobre el ambiente, subordinadas a la lógica del sistema patriarcal dominante. Vale la pena recordar algunos de sus planteamientos, por cuanto se constituyen en vías de acceso para resolver problemas de carácter estructural, como la pobreza, la desigualdad y la exclusión social, y, con estos, la falta de participación de las mujeres en el ámbito público, así como una falta de reconocimiento sobre el respeto al medio ambiente.

* Este artículo es producto de la reflexión y avances que la autora ha venido desarrollando a partir de la investigación "Naturaleza y ambiente: dos categorías que se enfrentan", inscrito en la línea de investigación Comunicación, Salud, Medio Ambiente y Sostenibilidad.

** Magíster en Ciencias Sociales. Docente investigadora de la Universidad Santo Tomás. Correo electrónico: yanethortiz@usantotomas.edu.co 
Precisamente la naturaleza ha sido un tema de preocupación para diversos actores, por su relación con los seres humanos. La falta de conciencia sobre el estado de depredación de los recursos biofísicos encuentra su razón de ser en un sistema capitalista que enseña el dominio sobre la naturaleza y su uso instrumental, contrario a la lógica de las culturas ancestrales, así como de los nuevos movimientos sociales, que optan por una relación de respeto con ella, de interdependencia. De manera especial, este artículo se plantea diferentes perspectivas en torno al ecofeminismo, por cuanto las categorías mujer y naturaleza son temas que se han ido posicionando en el ámbito local y global, como parte de la agenda de los gobiernos y las organizaciones, y como una apuesta política de las comunidades.

\section{Principales tendencias que configuran el ecofeminismo}

La primera en utilizar el término ecofeminismo fue Françoise d'Eaubonne, en 1974, quien buscaba representar el potencial de las mujeres para encabezar una revolución ecológica que conllevara a nuevas relaciones de género entre hombres y mujeres, y a una relación distinta entre los seres humanos y la naturaleza (Olarte, 2006, p. 18). Si bien existen tantos ecofeminismos como teóricas ecofeministas, el término hace referencia a ese acercamiento y articulación de aportaciones del feminismo y del ecologismo.

Desde su aparición, el ecofeminismo ha desarrollado un extenso debate sobre las afirmaciones de las ecofeministas, que defienden la existencia de una conexión directa entre la dominación patriarcal de la naturaleza y de la mujer. Esto se ha descrito bien como una afinidad entre la mujer y la naturaleza, y como una experiencia compartida de dominación (Griffin; Collard; Plant, citados en Velayos, Barrios, Figuerelo y López, 2007). A partir de esto, es posible plantear el vínculo entre la mujer y la naturaleza en el contexto de una sociedad patriarcal, sin llegar a caer en los determinismos, porque lo que interesa analizar es el papel de las mujeres y su participación en la economía de mercado.

Feminismo y ecologismo serán dos movimientos sociales fundamentales en el siglo XXI, ante la insostenibilidad del actual modelo de desarrollo, centrado en el crecimiento, mas no en el equilibrio. Asimismo, el ecofeminismo, con sus diversas tendencias, será de vital importancia para dar un giro a las actuales concepciones de la realidad, a fin de preocuparse e interesarse por el cambio de las relaciones entre las personas y el medio ambiente, la igualdad de derechos y la abolición de las jerarquías (Olarte, 2006, p. 18).

El ecofeminismo ha señalado la importancia de reconocer la relevancia de sus tendencias para comprender las relaciones entre el feminismo y el medio ambiente, las cuales han sido estudiadas y perfectamente documentadas a lo largo de los años, tanto desde el punto de vista histórico, como del político, epistemológico 
y simbólico, por nombrar solo algunos de ellos. Las siguientes son perspectivas desde las cuales pretendo mostrar cómo se producen estos discursos, a quiénes reivindican y cuál es el lugar que ocupa la naturaleza.

1. Histórica. Algunas ecofeministas usan este enfoque para generar teorías sobre el origen de las dominaciones de la mujer y la naturaleza, y estudian cómo los cambios culturales y científicos ocurridos en la revolución industrial se orientaron en favor de la explotación de la naturaleza, la expansión del comercio y la dominación de la mujer.

2. Conceptual. Filósofas ecológico-feministas han argumentado que en última instancia, los vínculos históricos y causales entre la dominación de la mujer y la naturaleza se basan en la forma en que conceptualmente se han concebido. En este caso, los análisis se han dado bajo un marco interpretativo de la realidad opresivo patriarcal que explica, justifica y mantiene la subordinación de las mujeres hacia los hombres, caracterizado no solo por dualismos y jerarquías de valor (razón/emoción, mente/cuerpo, cultura/naturaleza), sino también por conceptos de poder particulares y una lógica de dominación que se explica desde que la superioridad justifica la dominación (Warren, 1996, p. 15).

3. Empírica. Recurre a aquellos datos empíricos - como la presencia de pesticidas y tóxicos, y la producción intensiva ganadera - ligados a conceptos y prácticas patriarcales, para explicar las relaciones de dominación y presentar la necesidad de plantear otros análisis críticos feministas -desde el ecologismo, el feminismo y la ética medioambiental - en pro de la preservación del ambiente, a modo de contribución para la creación de una postura global que defienda el principio de la vida.

4. Epistemológica. Las conexiones históricas, conceptuales y empíricas vinculan el feminismo con la naturaleza y revelan la necesidad de desarrollar epistemologías ecologistas y feministas diferentes; algunas parten de ideas propuestas por la Escuela de Frankfurt, ya que ofrecen una crítica a la naturaleza vs. la cultura y el andamiaje epistemológico necesario para analizar la relación entre la dominación de la mujer y la dominación de la naturaleza.

5. Simbólica. Algunos exploran la devaluación simbólica de la mujer y la naturaleza en el arte, la literatura y la teología; otros analizan el lenguaje sexista y el naturalista como lenguajes que subordinan $y$, a su vez, perpetúan la dominación e inferiorización de la mujer y la naturaleza. Por ello, uno de los propósitos de las ecofeministas al respecto es plantear una teoría y práctica feminista que no perpetúe este lenguaje ni los sistemas de poder que sustenta (Warren, 1996, p. 20). 
6. Ética. Esta tendencia observa la manera en que se plantea el ecofeminismo y la manera en que se trata la naturaleza y a las mujeres, lo cual requiere análisis éticos y feministas que involucren teorías y prácticas alejadas de una lógica patriarcal.

7. Teoréticas. Este enfoque incorpora las posturas de la ética consecuencialista, deontológica, y también la ecología social, la ecología profunda y la ética del cuidado, entre otras que abogan por crear éticas basadas en el respeto hacia la mujer y la naturaleza.

8. Política. La diversidad de perspectivas teóricas feministas que se preocupan por el medio ambiente es un reflejo de la posición política que desarrolla análisis sobre la dominación que explica, aclara y guía el activismo político. Como lo plantea Velayos (2007),

La mujer y la naturaleza no se quedaron fuera de los cálculos económicos por casualidad. No se trata de un error o de una injusticia que pueda paliarse con reformas adecuadas, como ya hemos visto. La exclusión de la mujer y de la naturaleza constituye un elemento central en la construcción social del hombre económico y del sistema económico que aquel representa. (p. 42)

Respecto a la discusión sobre la relación mujer-naturaleza, Warren (citado en Tardón, 2011) plantea cuatro perspectivas conceptuales desde el modo en que el binomio mujer-naturaleza ha sido catalogado en la tradición intelectual, regida muchas veces por los dualismos organizados jerárquicamente.

1. Los dualismos. El dualismo más conocido y generalizado es el de mente/ cuerpo: mientras que los hombres hacen mejorar la civilización, la función de las mujeres se basa en mejorar la especie. El dualismo producción/reproducción ha instalado no solo diferencias entre el hombre y la mujer, sino también claras desventajas respecto a su papel en la sociedad. El trabajo reproductivo y de cuidado se desarrolla casi de manera exclusiva en el ámbito doméstico, en el que, en gran parte, se carece de remuneración económica. Esta lógica de división sexual del trabajo genera, especialmente en las mujeres, la imposibilidad para ser independientes y autónomas, y limita su participación en otros escenarios. Al respecto, Bonder (1998) afirma que este binarismo es expresión de un imaginario masculino, devenido en discurso científico, en el que la naturaleza ha sido representada como un espacio vacío, inerte, 'femenino', dispuesto a ser penetrado por la inscripción cultural 'masculina'. Este tratamiento sería parte de una práctica regulatoria que produce los cuerpos de varones y mujeres como diferentes y complementarios, bajo una lógica que asume la heterosexualidad como norma. En este sentido, el sexo, lejos de ser algo dado o presimbólico, es, en su opinión, una categoría política. "El sexo no es lo que uno es, sino en lo que uno se convierte" (Bonder, 1998). 
Por lo demás, este dualismo de lo biológico y lo cultural no sería más que otra expresión de una lógica binaria que funda y legitima ordenamientos jerárquicos al oponer hombre y mujer, cuerpo y espíritu o psique, razón y emoción, entre otros.

2. Los conceptos de poder. Parecen ser siempre vistos desde la dominación, la cual viene respaldada por una serie de justificaciones que favorecen la superioridad masculina sobre la mujer (Tardón, 2011). El papel del ecofeminismo se orienta entonces a fragmentar estas lógicas de poder, no solo con los grupos humanos, sino también con el ambiente. No hay que olvidar que cuando aparece la propiedad privada, la naturaleza empieza a ser vista como un recurso que hay que usufructuar, más que una totalidad sin la cual no sería posible la vida.

3. Ética del medioambiente y organización de la economía. Este dualismo propone la combinación de feminismo y ética medioambiental como creador de lenguajes y prácticas que tienen en cuenta el género, sin promover la explotación en aquellos ámbitos por lo general identificados con lo masculino. En esta relación entre ética medioambiental y feminismo, las mujeres, históricamente, han sido las responsables del cuidado y la conservación del ambiente, lo que hace patente el vínculo entre las mujeres y la naturaleza con la economía de subsistencia que ellas llevan a cabo frente a la economía de mercado. Vandana Shiva focaliza la atención en un cambio de concepción que no tiene por qué ser utópico, y que se basa en un lema principal: un cambio de la economía del dinero por la economía de la naturaleza:

[...] si el producto primero fueran las materias primas (agua, tierra, etc.) y no el dinero, la perspectiva de la producción sería totalmente diferente y favorecería la vida de muchos agentes que se ven arruinados por no alcanzar los mínimos exigidos por el sistema. (Tardón, 2011, p. 8)

Esta lógica colonizadora, que irrespeta los ciclos naturales y no permite que la tierra se recupere, reafirma aquel planteamiento de que el principio femenino muere porque la tierra es concebida como materia prima.

1. Feminización de la naturaleza. En el cuarto estudio conceptual, Warren establece una comparación ente lo orgánico y lo científico respecto a la manera en que era concebida la naturaleza. La feminización de la naturaleza y la naturalización de la mujer, dos metáforas que tras la revolución científica, han perjudicado tanto a la una como a la otra. La naturaleza se ha convertido en ese ser vulnerable del que se puede abusar; la mujer, por su parte, ha sufrido las consecuencias de esa mecanización de lo orgánico, y ha quedado relegada al cuidado de los otros y de lo "otro", es decir, a la naturaleza, que en un sistema de mercado es 
una actividad poco valorada social y económicamente. Esta feminización de la naturaleza se viene usando no para rescatar los valores de la igualdad y la justicia, sino para explotarla de manera desmedida. Esta última propuesta invita a ambientalistas y a feministas a trabajar de manera conjunta en estrategias que hagan frente a las devastadoras tendencias mercantilistas.

Entre los enfoques del ecofeminismo, el radical, el liberal y el socialista coinciden en la existencia de un llamado a dominar y a explotar, tanto a los sujetos, como la naturaleza. El ecofeminismo radical recupera los valores matriarcales y la cultura femenina, y promueve la idea de que el papel de las mujeres en la preservación de la especie es un elemento fundamental de poder para ellas y un activismo político ecológico propio.

Por su parte, el ecofeminismo liberal se basa en el feminismo de la igualdad y la teoría conservacionista de la naturaleza. Sostiene que la crisis ambiental es el resultado de un esquema de desarrollo económico que olvida los impactos negativos que causa sobre el ambiente, no utiliza adecuadamente el patrimonio natural y no cuenta con una legislación al respecto. Plantea también que "la explotación de las mujeres es el resultado de la situación marginal en la que se les mantiene, pero la conexión entre mujeres y medio ambiente no tiene una base biológica" (Olarte, 2006, p. 19). Este pensamiento ecofeminista cuestiona tanto a mujeres como a hombres, quienes, al ser parte de una sociedad patriarcal, asumen ciertas actitudes que resultan no ser respetuosas con la naturaleza.

Otro de los enfoques es el ecofeminismo socialista, que considera que los problemas ambientales se deben al patriarcado y al capitalismo, que justifica la explotación de la naturaleza con el pretexto de facilitar el desarrollo y el crecimiento económico. El capitalismo ha alejado a los hombres de la naturaleza, les ha hecho pensar que son dueños de ella, y les ha brindado los medios para utilizarla y controlarla para su beneficio; asimismo, ha explotado a las mujeres, al subordinar su participación histórica en la economía (Olarte, 2006, p. 20).

Debido al olvido de las consecuencias de tal dominio sobre la vida de las mujeres, como la ausencia del poder para participar en asuntos comunitarios y la vulnerabilidad social, económica y cultural a la que se ven sometidas muchas de ellas, surge, con Bina Agarwal, un feminismo ecologista. Como una propuesta alternativa al ecofeminismo radical, plantea una alternativa a la crisis de los valores de la sociedad consumista e individualista actual. Según este enfoque, la relación de las mujeres con la naturaleza será diferente en virtud de factores como la clase social a la que pertenecen. Estos factores también determinan su relación con la organización de la producción, la reproducción y la distribución (Olarte, 2006, p. 20). Para Llort (1994), 
[...] desde el feminismo se debe desafiar y transformar el sistema de géneros, la división sexual del trabajo y la distribución desigual de los recursos entre los géneros. Desde el ecologismo se debe desafiar y cambiar las relaciones entre las personas y el medio ambiente, así como acabar con los procesos que permiten que una minoría se apropie de los recursos de la naturaleza. (p. 7)

Por su parte, el ecofeminismo crítico reivindica el principio de igualdad y la ética del cuidado, no solo con lo humano, sino también en la relación de lo humano con lo no humano; así, abre un espacio para pensar las relaciones de carácter intercultural y la posibilidad de construir un escenario cultural-ecológico. Puleo (2008) cuestiona la globalización capitalista: se manifiesta en contra de la desigualdad social y propone unas "claves teóricas y prácticas para labrar un futuro más sostenible" (p. 48). Defiende su afirmación de que otro mundo es posible, mientras reflexiona sobre el significado de conceptos como libertad, igualdad y sostenibilidad, reflexión no solo ética, sino también sociopolítica, sobre las relaciones de los seres humanos con la naturaleza.

La propuesta de ecofeminismo crítico que propone Puleo asume el concepto de género desde una mirada descriptiva y crítica, es decir, como categoría de análisis, para comprender los procesos históricos y culturales que construyen las identidades sexuadas, y para hacer visibles la desigualdad, la discriminación y los prejuicios de sexo, siempre que se les otorgue un contenido crítico. Este ecofeminismo crítico pretende señalar

[...] la necesidad de conservar, debidamente revisado y actualizado, el legado ilustrado de lucha contra el prejuicio de igualdad y autonomía de las mujeres [...] llamo a un diálogo intercultural, en especial con las culturas latinoamericanas, propongo una aceptación prudente de la ciencia y la técnica, una universalización de las virtudes del cuidado aplicadas a los humanos y al resto de la Naturaleza (universalizar en el sentido de enseñar las actitudes del cuidado a los varones y no esperarlas solo de las mujeres) y una ética de la justicia y la compasión más allá de nuestra especie. (Puleo, 2008, p. 49)

Dicha apuesta busca superar esa perspectiva estereotipada de la mujer como una víctima y, por el contrario, ubica la posibilidad que tienen mujeres y hombres de generar procesos de cambio y transformación social.

Desde el ecologismo, se concibe la realidad a partir de una perspectiva holística, para entender que estamos en un sistema en el que todos los elementos están interrelacionados, y en el que dependemos unos de otros. El feminismo brinda una visión que incorpora lo femenino, y muestra otras formas de relacionarse con la naturaleza mediante el cuidado. El ecofeminismo nos permite tomar distancia respecto a la sociedad consumista, devastadora e individualista que nos rodea; así, se plantea como un ecologismo social, que se pregunta por las conexiones 
existentes entre la explotación que se ejerce sobre la naturaleza y la que sufren los seres humanos.

Si bien el ecofeminismo es la conciencia de género de la ecología política, esto no significa que sea una sección especial de aquella, ni que se subsuma en ella. El ecofeminismo, como el feminismo en general, es múltiple, y conviene que conserve su independencia y su diversidad (Puleo, 2008). Es interesante cómo muchas personas, mediante sus prácticas cotidianas, apoyan ciertas formas de activismo ecológico, aunque no se denominen ecofeministas. En un sentido más amplio, hasta las tendencias del ciberactivismo y del ciberfeminismo pueden considerarse ecofeministas, si se acompañan de una visión no sexista, y llevan a la creación de una nueva cultura de igualdad y sostenibilidad.

Detenernos en esta cuestión nos parece fundamental para clarificar no solo nuestra producción teórica, sino también nuestras prácticas. Después de todo, cada vez que enunciamos y realizamos alguna acción dirigida a revertir la discriminación o subordinación de las mujeres, estamos sosteniendo, consciente o inadvertidamente, alguna concepción sobre el sujeto de esta acción, le asignamos determinadas características, modos de "funcionar", y suponemos, aunque pocas veces lo hagamos explícito, que será afectada/o de alguna manera particular por la intervención de la que se trate. Por lo demás, y como lo señala Braidotti, la problematización sobre la mujer, lo femenino, e incluso el género, está inextrincablemente vinculada a la crítica y deconstrucción del sujeto racional, universal moderno. (Bonder, 1998, p. 6)

Volviendo a la perspectiva ética de Shiva, el ecofeminismo plantea la necesidad de generar espacios de libertad, donde la diversidad y la autonomía sean los valores que guíen las acciones de los hombres y mujeres para la construcción de una sociedad sostenible. El reconocimiento de la igualdad de todos ante la ley, pero especialmente de la igualdad de oportunidades que garantice o cree las condiciones para la participación plena, debería ser el principio rector no solo para el reconocimiento de las diferencias, sino también del respeto que merece el otro, sus espacios y sus acciones (Shiva, citado en Santana, s. f.)

La propuesta de Shiva se distancia de un ecofeminismo clásico, por cuanto considera que para reivindicar el espacio de participación, no es necesario partir de una división entre los ámbitos público y privado —este último, asignado culturalmente a las mujeres y considerado algo insignificante e inferior, contrario al mundo de los hombres, configurado más creativo, productivo y generador de conocimiento y desarrollo.

"La perspectiva ecofeminista ve en los patrones culturales y simbólicos capitalistas patriarcales los soportes mediantes los cuales se ha justificado la explotación tanto de la naturaleza como de la mujer" (Santana, s. f.). De esta manera, la postura patriarcal refiere como "natural e inevitable" la dominación y 
explotación de la tierra, los animales y la mujer. Esta disputa por la recuperación de la naturaleza se ha asumido desde la década de los noventa, en especial por algunos movimientos ambientalistas de mujeres, quienes se han preocupado por vincularse a la discusión en cumbres, foros y congresos, para sentar su voz de protesta respecto a la tendencia destructiva que ha producido la racionalización e instrumentalización del sistema de mercado capitalista neoliberal.

En consecuencia, ante el evidente deterioro ambiental, activistas y teóricas feministas latinoamericanas plantean la necesidad de pensar en un nuevo orden mundial: un orden que dé prioridad a una política de desarrollo sustentable, y que involucre a las mujeres de manera directa, para que se les conceda una mayor participación en la formulación, ejecución y evaluación de políticas y toma de decisiones sobre el uso, protección y rehabilitación de la naturaleza.

\section{La propuesta ecofeminista latinoamericana}

El ecofeminismo en América Latina surgió hace dos décadas, con representantes como Safina Newbery, en Argentina; Ivonne Gebara, en Brasil; Mary Judith Ress, en Chile; Rosa Dominga Trapazo, en Perú, y Graciela Pujol en Uruguay, entre otras, quienes vienen proporcionando, desde sus países, algunas respuestas a la actual crisis ecológica y su vinculación con el modelo capitalista neoliberal. Junmto con estas, otras mujeres y hombres están promoviendo en el ámbito local iniciativas de protección, recuperación y conservación del ambiente. Tal vez la más reconocida es Ivone Gebara, quién a partir de

[...] las experiencias concretas de mujeres pobres y marginadas, presenta una visión crítica de la antropología dualista, buscando pensar de un modo nuevo la antigua imagen de la Trinidad, considerándola una realidad inserta en el cosmos, en la tierra, en las relaciones entre los pueblos y culturas. (Santana, s. f.)

Gebara revisa la posibilidad de vincular la forma feminista de pensar la vida con la mirada ecológica, al sugerir no solo la posibilidad real de igualdad entre mujeres y hombres de diferentes culturas, sino una relación diferente entre nosotros, con la tierra y con todo el cosmos.

Dos conceptos son claves en su enfoque: experiencia y deconstrucción. La experiencia refleja el punto de partida de todas sus reflexiones; la deconstrucción consiste en la crítica a conceptos tradicionales, para luego reconstruir desde otro lugar, desde nuevas necesidades.

Tal vez estas repuestas tienen todavía poco impacto en lo que significa la intención de contribuir, desde el ecofeminismo, en la construcción de una 
nueva cultura planetaria, cuyo eje principal es la recuperación de la tierra y el mejoramiento del ambiente como elemento importante en la búsqueda de mayores niveles de calidad de vida. Otra contribución de las ecofeministas latinoamericanas radica en el hecho de estar llamando la atención para evitar caer en la trampa tendida por los organismos internacionales financiadores del desarrollo y promovedores del desarrollo sostenible, al pretender adjudicarles a las mujeres la responsabilidad del mejoramiento ambiental, catalogándolas como "salvadoras del planeta". El compromiso con la vida y con el futuro planetario debe ser compartido por todos, por hombres y mujeres de manera igualitaria. (Santana, s. f.)

El ecofeminismo latinoamericano ha sido influenciado por la deep ecology, y el feminismo (en América Latina) se ha vinculado a corrientes anteriores ya arraigadas: la filosofía y la teología de la liberación, pues sus temas se han conjugado alrededor de cuestiones relativas a la praxis concreta de la liberación. Esta propuesta de ecofeminismo asume del feminismo dos afirmaciones fundamentales: 1) que el género (entendido como rol que corresponde a cada sexo dentro de una determinada comunidad) es un constructo social y no se deriva necesariamente del sexo biológico; 2) en esta diferenciación de roles (sesgo de género), la primacía ha correspondido al hombre, que ha dominado a la mujer (patriarcado) y ha establecido los modos masculinos de comportamiento o pensamiento como modelos para toda la humanidad (androcentrismo). Es decir, el género no es una propiedad de los sujetos ni es un constructo fijo y terminado, lo que nos permite detectar y explicar cómo "los sujetos se generan en y a través de una red compleja de discursos, prácticas e institucionalidades, históricamente situadas, que le otorgan sentido y valor a la definición de sí mismos y de su realidad" (Bonder, 1998).

Las feministas de la década de los sesenta en Europa no alcanzaron a vislumbrar que sus demandas desconocían la relevancia del contexto social y económico en sus discursos; por ello, las feministas del sur critican esta postura, pues comprenden que sus experiencias de vida son diferentes de las experiencias de mujeres de clase media del primer mundo, y, por eso, orientaron el feminismo hacia otras direcciones, privilegiando el análisis de los problemas de las sociedades periféricas. De ahí que este ecofeminismo tome la misma orientación.

Un ejemplo de lo anterior es el feminismo poscolonial ${ }^{1}$, que pone de relieve algunos puntos, como la idea de que sobre las mujeres del sur existe una confluencia de opresiones que es fundamental para moldear su experiencia de marginalidad política y social:

1 Si bien algunas autoras optan por hablar de feminismo del tercer mundo, feminismo poscolonial parece preferible, porque daría cabida tanto a las mujeres oprimidas del primer mundo, como a las mujeres de los países neocolonizados. 
[...] el papel crucial que ha tenido y tiene el Estado hegemónico, que domina su vida diaria y su lucha por sobrevivir; el significado de la memoria y la escritura en la construcción de una oposición activa; las diferencias, conflictos y contradicciones internas a las organizaciones y comunidades del Tercer Mundo. (Portolés, 2004, p. 17)

El desarrollo del ecofeminismo latinoamericano, que aún es incipiente, presenta, sin embargo, dos perspectivas que permiten identificarlo con un perfil propio: la revaloración de las cosmovisiones autóctonas (antiguas) y el énfasis en la praxis de liberación (Lertora, s. f.). Esta corriente del ecofeminismo se perfila, por un lado, como una apuesta teórica y práctica, preocupada por los graves problemas ambientales presentes en la región, resultado de un mal desarrollo enfocado en la explotación de la naturaleza - que a su vez es hábitat de comunidades empobrecidas-, y, por la otra, como la necesaria crítica al esencializar a la mujer, e incluso al género femenino, como una categoría única, y muchas veces deshistorizada, que ignora la heterogeneidad de las mujeres dentro de la categoría mujer, y fundamentalmente la diversidad existente en cada una de ellas en tanto sujetos no unitarios, sino múltiples y fragmentados, en diversas posiciones sociales y étnicas (Bonder, 1998).

\section{A manera de conclusión}

Detenerse a examinar las distintas perspectivas ecofeministas plantea importantes diferencias. Algunas de ellas pueden explicarse por los intereses y la formación inicial de las pensadoras, puesto que ponen el énfasis en aquello que conocen bien. Por ejemplo, el profundo dualismo y jerarquización inherentes a los conceptos de naturaleza y cultura, mujer y hombre. Algunas feministas, por su parte, se han inclinado por un abordaje socioeconómico, gracias al cual han logrado hacer visible la similitud entre el trabajo doméstico no remunerado de las mujeres y los servicios de la naturaleza, que no son reconocidos. Activistas altermundialistas y anticolonialistas, como Vandana Shiva, ponen de relieve las injusticias que cometen los países del norte con los países del sur, y denuncian el 'mal desarrollo' exportado por las multinacionales, un mal desarrollo que causa miseria, pérdida de la biodiversidad y desaparición de la diversidad cultural, aplastada por el modelo único del sistema económico capitalista.

En cuanto al ecofeminismo latinoamericano, este cuenta con un perfil propio: el rescate de las cosmovisiones autóctonas (antiguas) y el énfasis en la praxis de liberación, es decir, en la visión de que las mujeres no son las exclusivas salvadoras de la naturaleza, sino también los hombres. Se trata de contribuir a un cambio 
que permita que las prácticas de cuidado se universalicen y que sean hombres y mujeres quienes se ocupen de salvaguardar el mundo natural.

Al considerar las reflexiones sobre las perspectivas ecofeministas, es necesario preguntarse: ¿no será que el problema actual es más bien el resultado de una mirada que cosifica la naturaleza y prioriza un sistema económico? El problema está en ciertas formas de ser y hacer en el mundo, en una racionalidad instrumental que va generando diferentes tipos de realidades, cuyo proceso degrada o destruye toda forma de existencia, en desconocer el vínculo entre mujer y naturaleza, su capacidad creativa y creadora en el ámbito local y global, como parte de la agenda de las organizaciones, y como una apuesta política desde las comunidades.

\section{Referencias}

Bonder, G. (1998). Género y subjetividad: avatares de una relación no evidente. Recuperado de http://www.iin.oea.org/IIN/cad/actualizacion/pdf/ Explotacion/genero_y_subjetividad_bonder.pdf

Gargallo, F. (S. F.) Las ideas feministas latinoamericanas. Recuperado de http:// pmayobre.webs.uvigo.es/descargar_libros/las\%20ideas\%20feministas\%20latinoamericanas.pdf

Lértora, C. (S. F.) Ecofeminismo latinoamericano. Recuperado de http://www.cecies.org/articulo.asp?id=387

Llort, I. (1994). Ecofeminismo(s) o feminismo ecologista. Estado del debate en la India. Recuperado de http://www.geocities.com/equipasia/Art_ Ecofeminismo_Imma.htm

Olarte, A. (2006). Perspectiva ecofemenina de la dirección empresarial: una visión compleja. Recuperado de http://www.bdigital.unal.edu.co/1181/1/anacarolinaolartegrajales.2006.pdf

Portolés, A. (2004). Feminismo postcolonial: la crítica al eurocentrismo del feminismo occidental. Cuadernos de Trabajo, (6). Recuperado de pendientedemigracion.ucm.es/info/instifem/.../cuaderno\%206.doc

Puleo, A. (2008). Libertad, igualdad, sostenibilidad. Por un ecofeminismo ilustrado. Recuperado de http://www.fuhem.es/media/cdv/file/biblioteca/ Bolet\%C3\%ADn\%20ECOS/Boletin_10/Libertad_igualdad_sostenibilidad.pdf

Santana, N. (S. F.). El ecofeminismo latinoamericano. Las mujeres y la naturaleza como símbolos. Recuperado de http://www.saber.ula.ve/bitstream/123456789/18839/2/articulo5.pdf 
Tardón, M. (2011). Ecofeminismo. Una reivindicación de la mujer y la naturaleza. Recuperado de file:///C:/Users/usuario/Downloads/Dialnet-Ecofemin ismoUnaReivindicacionDeLaMujerYLaNaturalez-3697663\%20(1).pdf

Velayos, C., Barrios, O., Figuerelo, A. y López, T. (2007). Feminismo ecológico. Estudios multidisciplinares de género. Salamanca: Universidad de Salamanca.

Warren, K. (1996). Filosofías ecofeministas. Barcelona: Icaria. 
